\title{
MULTI-LEVEL DYNAMIC MODELING IN BIOLOGICAL SYSTEMS Application of Hybrid Petri Nets to Network Simulation
}

\author{
Rafael S. Costa ${ }^{1,3}$, Daniel Machado ${ }^{1}$, A. R. Neves ${ }^{2}$ and Susana Vinga $a^{3,4}$ \\ ${ }^{1}$ IBB-Institute for Biotechnology and Bioengineering, University of Minho, Campus de Gualtar, 4710-057 Braga, Portugal \\ ${ }^{2}$ ITQB/UNL, R. Qta Grande 6, 2781-901 Oeiras, Portugal \\ ${ }^{3}$ Instituto de Engenharia de Sistemas e Computadores, Investigação e Desenvolvimento (INESC-ID), \\ R. Alves Redol 9, 1000-029 Lisboa, Portugal \\ ${ }^{4}$ FCM-UNL, C. Martires Pátria 130, 1169-056 Lisboa, Portugal
}

\begin{abstract}
Keywords: Systems biology, Integrated dynamic modelling, Hybrid Petri net, Approximate rate laws, Streptococcus pneumoniae.

Abstract: $\quad$ The recent progress in the high-throughput experimental technologies allows the reconstruction of many biological networks and to evaluate changes in proteins, genes and metabolites levels in different conditions. On the other hand, computational models, when complemented with regulatory information, can be used to predict the phenotype of an organism under different genetic and environmental conditions. These computational methods can be used for example to identify molecular targets capable of inactivating a bacterium and to understand its virulence factors. This work proposes a hybrid metabolic-regulatory Petri net approach that is based on the combination of approximate enzyme-kinetic rate laws and Petri nets. A prototypic network model is used as a test-case to illustrate the application of these concepts in Systems Biology.
\end{abstract}

\section{INTRODUCTION}

Living cell systems are complex networks with different kinds of interacting chemical compounds (e.g. genes, small molecules and proteins). The interactions with each other propagate through the global network and the understanding of how a system behavior emerges is a major aim of the recent field of science called Systems Biology (Kitano, 2002). Understanding the mechanisms of the cell is essential for research in several areas such as drug design with applications to personalized medicine (Zhu et al., 2008), identification potential drug targets (Lee et al., 2009) and metabolic engineering (Park et al., 2007).

By using mathematical models of cellular metabolism, it is possible to systematically test and predict the optimal environmental and genetic manipulations (Di Ventura et al., 2006). The availability of genome sequences for many microorganisms has allowed the development of constraint-based models representing the global network of metabolic reactions (Price et al., 2003). However, while capturing most mass balance effects, those models ignore regulation and cannot be used for dynamic simulations. Moreover, applying constraint based methods directly to integrated networks is challenging (Covert et al., 2008). Ideally, the models would comprise different levels of knowledge, from reactions stoichiometry to reactions kinetics and regulatory information (e.g. transcriptional and enzymatic regulation) (Klipp, 2007). This is the basis of the E-Cell project (Tomita et al., 1999), that uses an approach to represent the dynamic state inside the whole cell. Although until recently most cellular components have been studied individually, the behavior of the cell emerges at the network-level interactions and requires an integrative analysis. Several models and analysis focused primarily on isolated network models rather than integrated systems. Examples of this are the analysis of signaling (Hoffmann et al., 2002), metabolic (Chassagnole et al., 2002) and regulation (Lee et al., 2002) systems. Thus, it is highly desirable that information obtained from genome annotation, together with kinetic and regulatory information can be used for the construction of integrated models that account for various interactions at diverse temporal and spatial scales.

Petri nets are a mathematical formalism that have been applied in the modeling of several biological pathway systems (Chaouiya et al., 2004; Chen et al., 2007; Zevedei-Oancea et al., 2003). Hybrid Petri nets are a particular type of Petri net extension devel- 
oped to accomodate discrete and continuous elements within the same formalism. This makes them specially appealing for the integration of gene regulatory and metabolic networks, that are more commonly represented as Boolean networks and differential equations, respectively. This idea was explored in (Chen and Hofestadt, 2003) to build a regulated metabolic model of the urea cycle, and also in (Doi et al., 2004) to reconstruct the glycolytic pathway controlled by the lac operon.

The recent advent of so-called genome-wide highthroughput methods have generated the called omics data (e.g. metabolomics, proteomics, transcriptomics and genomics) of bio-molecules like mRNA, proteins and metabolites, the detection of molecular interactions, such as, protein-DNA and protein-metabolite (Nielsen and Olsson, 2002). This has allowed a better understanding of the regulation of metabolism at a global scale (Lee et al., 2005). However, in spite of the great advances in the area, we are still far from a whole-cell computational model that integrates different omics data, as there exists a few approaches for the model-driven interpretation of these data and models that couple enzymatic and transcriptional regulation (Bettenbrock et al., 2006; Kotte et al., 2010; Tenazinha and Vinga, 2011; Usuda et al., 2010; Patil and Nielsen, 2005).

The main aim of this work is the creation of an integrating framework for metabolic and regulatory modeling of biological systems, including how we address some challenges in integrated systems. This paper is organized as follows: Section 1 explores the state of the art and the motivation for the work; Section 2 presents a hybrid kinetic modeling framework and Section 3 discusses their application to a prototypic small network. Section 4 discusses remaining challenges and our future directions.

\section{METHODS}

\subsection{Model Topology}

In order to evaluate the proposed approach we used as an example the model topology shown in Figure 1. It comprises some of the glycolysis reactions for Streptococcus pneumoniae retrieved from the draft model reconstruction using the SEED web-tool (Henry et al., 2010) and information from KEGG (Ogata et al., 1999) database. The biochemical interactions of these reactions have been translated into the Petri net model used in this work. The network consists of 5 continuous transitions representing the reactions; 8 continuous places representing 8 metabolites; and 2 discrete places and 2 discrete transitions were used to create the regulatory components (on/off switch).

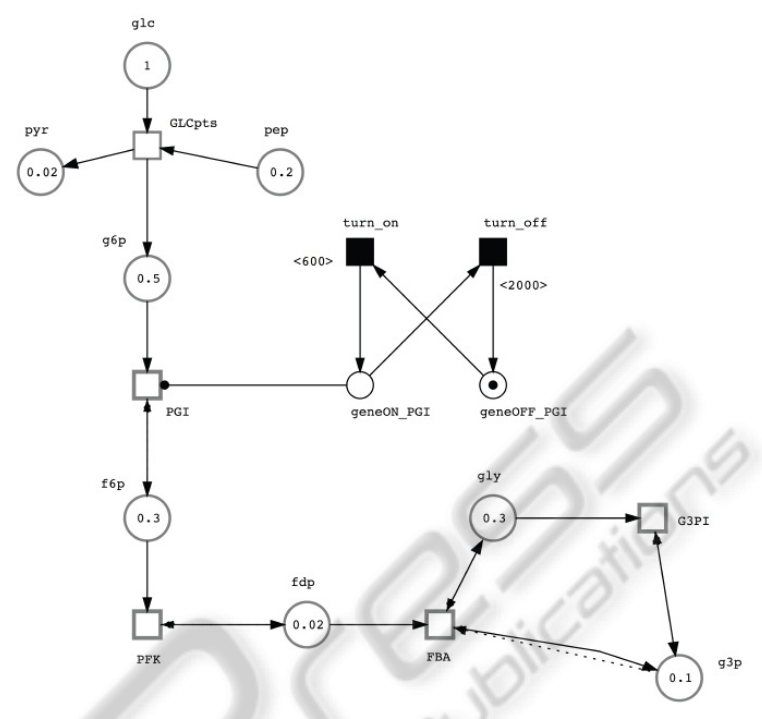

Figure 1: Hybrid Petri net representation of the prototypic metabolic-regulatory network buit with the Snoopy sofware tool (Rohr et al., 2010). The metabolites are represented by circles (places) and the enzymes by squares (transitions). Metabolites abbreviations: pyr, pyruvate; pep, phosphoenolpyruvate; glc, glucose; g6p, glucose-6-phosphate; f6p, fructose-6-phosphate; fdp, fructose-1,6-bisphosphate; gly, glycine; g3p, glucose-3-phosphate. Enzymes abbreviations: GLCpts, phosphotransferase of PTS system (EC 2.7.3.9); PGI, glucose-6-phospate isomerase (EC 5.3.1.9); PFK, phosphofructokinase (EC 2.7.1.11); FBA, fructosebisphosphate aldolase (EC 4.1.2.13); G3PI, triosephosphate isomerase (EC 5.3.1.1).

\subsection{Modeling Process}

The overall idea of the modeling method is depicted in Figure 2. After the model topology construction, it is necessary to translate into mathematical differential equations and introduce the control of regulatory events. In this study, we use an extension of the original formalism known as hybrid Petri nets (Hassane and David, 1998) that accounts for metabolite dynamics coupled gene regulation and enzyme kinetics to construct the hybrid metabolic-regulatory model. The metabolic network is represented with continuous places and continuous transitions. Genes are represented by discrete places. Each gene is represented by two places (on and off), which are connected to transitions that activate and inactivate the gene, ensuring that the activation of both places is mutually exclusive. The connection between both layers is made by means of activation arcs between the reactions and the respective genes. The Petri net model of the network was drawn using the graphical editor of the Petri 
net tool Snoopy (Rohr et al., 2010). Snoopy is a general purpose software application for design and simulation of graph-based formalisms and is freely available.

Recently, we have studied several types of approximate enzyme-kinetic rate laws (Costa et al., 2010). In this study, approximate kinetics lin-log representations are used for the rate equations, but alternatively other approximate rate laws (e.g convenience kinetics) can be also integrated. Lin-log kinetics (Visser and Heijnen, 2003) are formulated based on a reference rate $r^{0}$, and given by:

$$
r=r^{0} \frac{e}{e^{0}}\left(1+\sum_{i}^{n_{S}} \varepsilon_{S_{i}}^{0} \ln \left(\frac{S_{i}}{S_{i}^{0}}\right)+\sum_{j}^{n_{P}} \varepsilon_{P_{j}}^{0} \ln \left(\frac{P_{j}}{P_{j}^{0}}\right)\right)
$$

where the superscripts $\left({ }^{0}\right)$ denote the reference state (e.g. wild type at steady-state), $r^{0}$ is the reference reaction rate value, $e / e^{0}$ represent the relative enzyme activities, $S_{i} / S_{i}^{0}$ and $P_{j} / P_{j}^{0}$ are the relative concentrations that influence the kinetics of the reaction.

The negative of the corresponding stoichiometric coefficients were set as the initial guesses for the elasticities parameter values as suggested by (Smallbone et al., 2007). The initial concentrations of metabolites were taken as arbitrary values and are assuming that the reference steady-state is known $(\mathrm{pyr}=0.02$ $\mathrm{mM}$; pep =0.2 mM; glc, $=1 \mathrm{mM} ; \mathrm{g} 6 \mathrm{p}=0.5 \mathrm{mM}$; $\mathrm{f} 6 \mathrm{p}=0.3 \mathrm{mM} ; \mathrm{fdp}=0.02 \mathrm{mM} ; \mathrm{gly}=0.3 \mathrm{mM} ; \mathrm{g} 3 \mathrm{p}$ $=0.1 \mathrm{mM}$ ). The simulation process of the metabolicregulatory model performed in this study, were carried out using the Snoopy tool (Rohr et al., 2010).

\section{ILLUSTRATION OF A PROTOTYPIC NETWORK}

Computational models are very useful for exploring the effects of manipulating pathways through drugs. The aim of this study was to present an approach to construct a hybrid mathematical kinetic model that accounts for the metabolic and regulatory events. This approach was applied by taking the small prototypic integrated system of S. pneumoniae (shown in Figure 1) as an example.

In Figure $3 \mathrm{a}$ and $3 \mathrm{~b}$ simulation time courses of all the metabolite concentration show the effect of a gene activation with and without the regulation part, respectively.

We demonstrated how inclusion of regulatory events may affect the phenotype behavior. As an example of this changes, at time $=600$ seconds the $p g i$

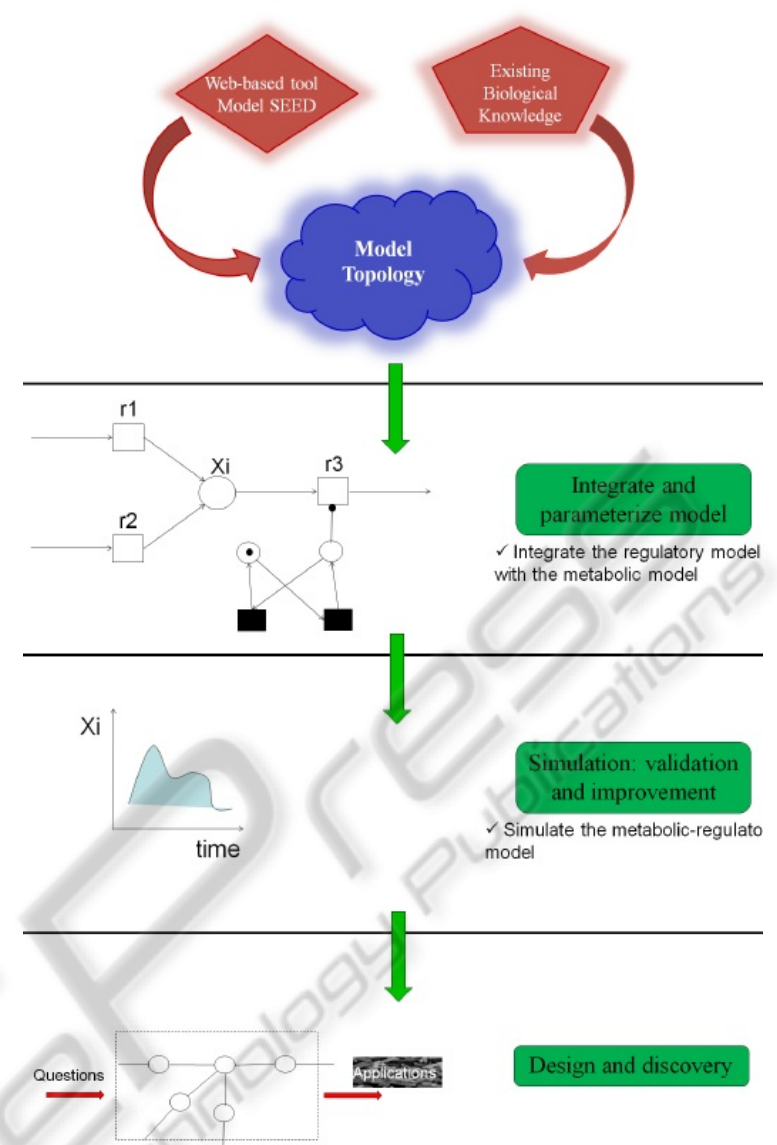

Figure 2: Overview of the modeling process from the construction of a biochemical reaction network to conversion into a mathematical format. (1) SEED web-based software is used to reconstruct the metabolic network including the metabolic part and the regulatory information are based on existing biological knowledge. (2) Conversion of the metabolic network to a computational model. Combining the regulatory events and the metabolic information in a hybrid Petri net modeling framework. (3) Modeling and simulation in Snoopy tool (Rohr et al., 2010). (4) Model application example: identify essential genes encoding new targets for antibacterial drugs that can be tested in vitro for reduction of pathogenicity.

gene is activated and the affect on the dynamical behaviour of the g6p, f6p, fdp, gly and g3p metabolites are shown, reaching a new steady state a time $=1100$ seconds (3a). Our results show that regulation has influence on the steady-state distribution obtained in comparison with the model without the regulation effect. Accounting for these regulations may have a significant impact in the phenotype predictions and can reveal new therapeutic targets. 

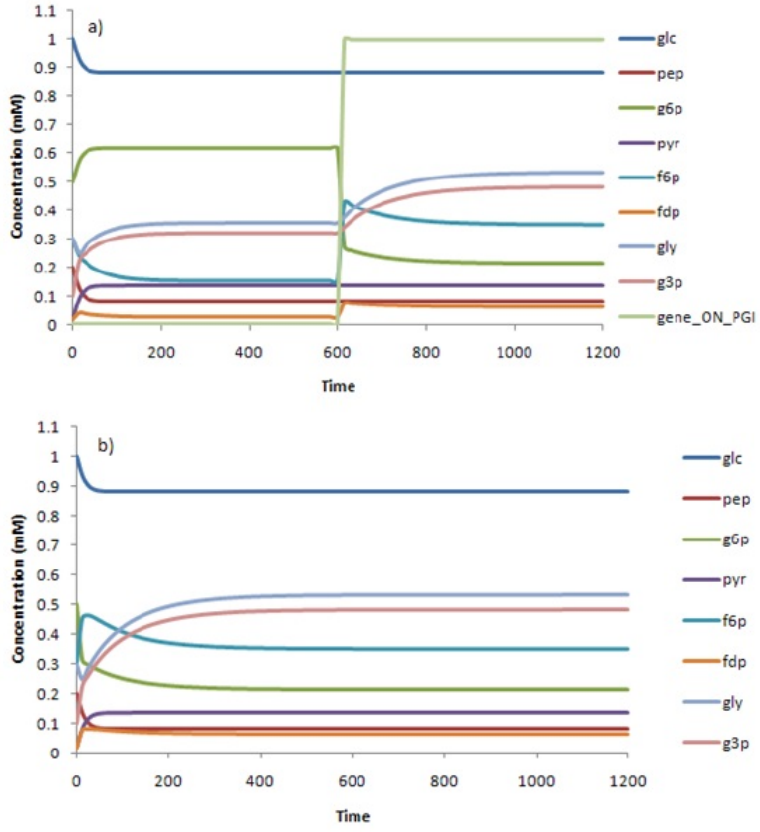

Figure 3: Simulation of the dynamics for the prototypic $S$. pneumoniae model with the pgi gene activation after $600 \mathrm{~s}$ (a) and without accounting regulatory effects (b). The integrated system, comprise metabolic and regulation events.

\section{CONCLUSIONS AND FUTURE WORK}

This work presents a strategy to build a hybrid metabolic-regulatory model based on a Petri net framework. This work addresses also the impact of accounting of a kind of regulation which comes from gene regulation. This approach has significant advantages, because the model design and analysis is easily connected between the continuous and discrete features that would otherwise be unforeseen. Moreover, it can reveal insights into disease mechanism and possible therapeutic targets with applications to health.

On the other hand, there are still some current problems in our proposed modeling approach that need to be addressed. The kinetic parameter values estimation is a significant bottleneck. Here we use the negative of the stoichiometry from each reaction for the parameters as suggested by (Smallbone et al., 2007). However a natural attempt is to distinguish kinetic parameters for the discrete and continuous events of the hybrid model. In order to estimate the kinetic parameters of the metabolic reactions, the proposed procedure is to isolate the metabolic network and perform parameter fitting using experimental data (metabolites and fluxes time course data) on a short time scale for which the genetic part can be considered constant. For this purpose, in the future it is intended to use experimental steady-state and timecourse metabolomic and fluxomic data from S. pneumoniae that will be generated by other participants in the PneumoSyS project. This experimental data will be also used for model validation. Another limitation is that the metabolic reconstructions obtained from the SEED tool do not account for the regulatory interactions. Therefore, it will be necessary to annotate the model topology reconstruction with such information, which can be obtained from available literature and web-databases. An additional challenge of integrated modeling approaches are time scales. For example, metabolic reactions occur on the order of seconds. By contrast, regulatory reactions can take several minutes to hours.

In future work, we intend develop and validate a complete dynamic hybrid metabolic-regulatory model of $S$. pneumoniae using the proposed approach to predict disease-related metabolic states, which is of major bio-medical interest.

\section{ACKNOWLEDGEMENTS}

This work was partially supported by post-doctoral grant by Fundação para a Ciência e a Tecnologia (FCT) (SFRH/BPD/80784/2011), project PneumoSyS - A Systems Biology approach to the role of pneumococcal carbon metabolism in colonization and invasive disease (FCT contract: PTDC/SAUMII/100964/2008) and by FCT (INESC-ID multiannual funding) through the PIDDAC Program funds.

\section{REFERENCES}

Bettenbrock, K., Fischer, S., Kremling, A., Jahreis, K., Sauter, T., and Gilles, E. (2006). A quantitative approach to catabolite repression in Escherichia coli. Journal of Biological Chemistry, 281:2578.

Chaouiya, C., Remy, E., Ruet, P., and Thieffry, D. (2004). Qualitative modelling of genetic networks: From logical regulatory graphs to standard Petri nets. Applications and Theory of Petri Nets 2004, pages 137-156.

Chassagnole, C., Noisommit-Rizzi, N., Schmid, J., Mauch, K., and Reuss, M. (2002). Dynamic modeling of the central carbon metabolism of Escherichia coli. Biotechnology and Bioengineering, 79(1):53-73.

Chen, L., Qi-Wei, G., Nakata, M., Matsuno, H., and Miyano, S. (2007). Modelling and simulation of signal transductions in an apoptosis pathway by using timed Petri nets. Journal of biosciences, 32(1):113127. 
Chen, M. and Hofestadt, R. (2003). Quantitative petri net model of gene regulated metabolic networks in the cell. In silico biology, 3(3):347-365.

Costa, R., Machado, D., Rocha, I., et al. (2010). Hybrid dynamic modeling of Escherichia coli central metabolic network combining Michaelis-Menten and approximate kinetic equations. Biosystems, 100(2):150-157.

Covert, M., Xiao, N., Chen, T., and Karr, J. (2008). Integrating metabolic, transcriptional regulatory and signal transduction models in Escherichia coli. Bioinformatics, 24(18):2044-2050.

Di Ventura, B., Lemerle, C., Michalodimitrakis, K., and Serrano, L. (2006). From in vivo to in silico biology and back. Nature, 443(7111):527-533.

Doi, A., Fujita, S., Matsuno, H., Nagasaki, M., and Miyano, S. (2004). Constructing biological pathway models with hybrid functional petri nets. In silico biology, 4(3):371-291.

Hassane, A. and David, R. (1998). Continuous and hybrid Petri nets. Journal of Circuits Systems and Computers, 8:159-188.

Henry, C., DeJongh, M., Best, A., Frybarger, P., Linsay, B., and Stevens, R. (2010). High-throughput generation, optimization and analysis of genome-scale metabolic models. Nature biotechnology, 28(9):977-982.

Hoffmann, A., Levchenko, A., Scott, M., and Baltimore, D. (2002). The ikb-nf- $k b$ signaling module: temporal control and selective gene activation. Science, 298(5596): 1241

Kitano, H. (2002). Systems biology: a brief overview. Science, 295(5560): 1662.

Klipp, E. (2007). Modelling dynamic processes in yeast. Yeast, 24(11):943-959.

Kotte, O., Zaugg, J., and Heinemann, M. (2010). Bacterial adaptation through distributed sensing of metabolic fluxes. Molecular Systems Biology, 6(1).

Lee, D., Saha, R., Yusufi, F., Park, W., and Karimi, I. (2009). Web-based applications for building, managing and analysing kinetic models of biological systems. Briefings in bioinformatics, 10(1):65-74.

Lee, S., Lee, D., and Kim, T. (2005). Systems biotechnology for strain improvement. TRENDS in Biotechnology, 23(7):349-358.

Lee, T., Rinaldi, N., Robert, F., Odom, D., Bar-Joseph, Z., Gerber, G., Hannett, N., Harbison, C., Thompson, C., Simon, I., et al. (2002). Transcriptional regulatory networks in Saccharomyces cerevisiae. Science, 298(5594):799.

Nielsen, J. and Olsson, L. (2002). An expanded role for microbial physiology in metabolic engineering and functional genomics: Moving towards systems biology 1 . FEMS yeast research, 2(2):175-181.

Ogata, H., Goto, S., Sato, K., Fujibuchi, W., Bono, H., and Kanehisa, M. (1999). KEGG: Kyoto encyclopedia of genes and genomes. Nucleic acids research, 27(1):29.

Park, J., Lee, K., Kim, T., and Lee, S. (2007). Metabolic engineering of Escherichia coli for the production of l-valine based on transcriptome analysis and in silico gene knockout simulation. Proceedings of the national academy of sciences of the USA, 104(19):7797.
Patil, K. and Nielsen, J. (2005). Uncovering transcriptional regulation of metabolism by using metabolic network topology. Proceedings of the national academy of sciences of the USA, 102(8):2685.

Price, N., Papin, J., Schilling, C., and Palsson, B. (2003). Genome-scale microbial in silico models: the constraints-based approach. TRENDS in Biotechnology, 21(4):162-169.

Rohr, C., Marwan, W., and Heiner, M. (2010). Snoopy - a unifying Petri net framework to investigate biomolecular networks. Bioinformatics, 26(7):974.

Smallbone, K., Simeonidis, E., Broomhead, D., and Kell, D. (2007). Something from nothing- bridging the gap between constraint-based and kinetic modelling. Febs Journal, 274(21):5576-5585.

Tenazinha, N. and Vinga, S. (2011). A survey on methods for modeling and analyzing integrated biological networks. IEEE/ACM Transactions on Computational Biology and Bioinformatics (TCBB), 8(4):943-958.

Tomita, M., Hashimoto, K., Takahashi, K., Shimizu, T., Matsuzaki, Y., Miyoshi, F., Saito, K., Tanida, S., Yugi, K., Venter, J., et al. (1999). E-CELL: software environment for whole-cell simulation. Bioinformatics, 15(1):72-84.

Usuda, Y., Nishio, Y., Iwatani, S., Van Dien, S., Imaizumi, A., Shimbo, K., Kageyama, N., Iwahata, D., Miyano, H., and Matsui, K. (2010). Dynamic modeling of Escherichia coli metabolic and regulatory systems for amino-acid production. Journal of biotechnology, 147(1):17-30.

Visser, D. and Heijnen, J. (2003). Dynamic simulation and metabolic re-design of a branched pathway using linlog kinetics. Metabolic Engineering, 5(3):164-176.

Zevedei-Oancea, I., Schuster, S., et al. (2003). Topological analysis of metabolic networks based on Petri net theory. In silico biology, 3(3):323-345.

Zhu, J., Zhang, B., and Schadt, E. (2008). A systems biology approach to drug discovery. Advances in genetics, 60:603-635. 